\title{
Estudio descriptivo del perfil neuropsicológico y psicopatológico en pacientes con distrofia miotónica tipo 1
}

Raquel Seijas-Gómez, Izaskun Basterra-Jiménez, Pilar Luna-Lario, Javier Tirapu-Ustárroz, Teresa Cabada-Giadás, Marina Iridoy-Zulet, Ivonne Jericó-Pascual, Álvaro Gargallo-Vaamonde, José Javier López-Goñi

Introducción. La distrofia miotónica tipo 1 (DM-1) o enfermedad de Steinert es un trastorno multisistémico y progresivo. Se han encontrado déficits cognitivos, clínica depresiva y alta incidencia de rasgos de personalidad ansiosos con afectación tanto en la funcionalidad como en la calidad de vida de estos pacientes.

Objetivo. Describir el perfil cognitivo y psicopatológico de una muestra de pacientes con la variante adulta de DM-1.

Pacientes y métodos. Se seleccionó una muestra de 27 pacientes con diagnóstico de DM-1 en seguimiento en el Servicio de Neurología del Complejo Hospitalario de Navarra. Los criterios de inclusión fueron tener menos de 50 años y descartar cualquier otra patología o condición física que impidiese realizar la evaluación psicológica. Se utilizó una batería de evaluación neuropsicológica específicamente diseñada para este tipo de patología, además de medidas de psicopatología y funcionalidad.

Resultados. La evaluación neuropsicológica reflejó, principalmente, déficits en habilidades visuoconstructivas, visuoespaciales, atención alternante y en sintomatología disejecutiva heteroinformada. El grupo de pacientes no presentó sintomatología depresiva ni ansiosa clínicamente significativa, pero sí puntuaciones elevadas en obsesión-compulsión, sensibilidad interpersonal, ideación paranoide y psicoticismo. Los resultados orientaron hacia un deterioro en la funcionalidad.

Conclusiones. En el abordaje integral de la DM-1, la caracterización y el seguimiento evolutivo del perfil cognitivo, psicopatológico y de personalidad, así como del nivel de funcionalidad, contribuyen a la mejora de la calidad de vida de estos pacientes.

Palabras clave. Distrofia miotónica. Enfermedad de Steinert. Estado emocional. Funcionamiento cognitivo. Personalidad. Síndrome disejecutivo.

\section{Introducción}

La distrofia miotónica tipo 1 (DM-1) o enfermedad de Steinert es la más frecuente de las distrofias musculares de aparición en la edad adulta [1]. Es una enfermedad genética de herencia autosómica dominante, asociada a la repetición anormalmente elevada del triplete CTG del 3'UTR del cromosoma 19q13.3 en el locus genómico de la cinasa denominada DMPK [2]. El número de repeticiones se correlaciona con la gravedad de los síntomas y con la edad de aparición de la enfermedad. El mecanismo molecular patogénico se relaciona con la ganancia de función tóxica del ARN anormalmente almacenado en los núcleos celulares y el splicing aberrante que esto induce en diferentes genes y que será responsable de las alteraciones multisistémicas que presentan estos pacientes [2]. La DM-1 es una enfermedad multisistémica con clínica muy variable. Aunque los síntomas principales afectan al músculo, también puede existir afectación cardíaca, oftálmica, gastrointestinal, endocrina y del sistema nervioso central. Por ello, es fundamental el estudio y seguimiento de estos pacientes de manera multidisciplinar, donde se incluya una evaluación neuropsicológica exhaustiva [2].

Los primeros estudios sistemáticos para caracterizar el perfil cognitivo y de personalidad se llevaron a cabo a partir de los años ochenta [3], con resultados inconsistentes. En la forma congénita es frecuente el retraso mental, leve o moderado, mientras que en la variante de inicio adulto son habituales otros síntomas cognitivos [4]. Se han descrito alteraciones en habilidades visuoespaciales y visuoconstructivas [5-11], ejecutivas, en razonamiento abstracto verbal [5-15], atención, memoria visuoespacial [7], facial [1] y verbal a corto y largo plazo [5], funcionamiento lingüístico $[8,11,16]$ y cognición social [17-19], y déficits relacionados con las medidas de personalidad, comportamiento, emociones y fun-
Área de Neuropsicología, Neuropsiquiatría y Terapia Ocupacional; Complejo Hospitalario de NavarraFundación Argibide (R. Seijas-Cómez, I. Basterra-Jiménez, P. Luna-Lario, J. Tirapu-Ustárroz). Servicio de Radiología (T. Cabada-Giadás) Servicio de Neurología (M. IridoyZulet, I. Jericó-Pascual, A. GargalloVaamonde); Complejo Hospitalario de Navarra. Universidad Pública de Navarra (J.J. López-Goñi). Pamplona, Navarra, España.

Correspondencia:

Dra. Raquel Seijas Cómez. Área de Neuropsicología, Neuropsiquiatría y Terapia Ocupacional. Complejo Hospitalario de Navarra. Antigua Clínica Ubarmin. E-31486 Elcano (Navarra).

E-mail:

raquelseijasgomez@gmail.com

Aceptado tras revisión externa: 03.09.15.

Cómo citar este artículo: Seijas-Cómez R, Basterra-Jiménez I, Luna-Lario P, Tirapu-Ustárroz J, Cabada-Giadás T, Iridoy-Zulet M, et al. Estudio descriptivo del perfil neuropsicológico y psicopatológico en pacientes con distrofia miotónica tipo 1. Rev Neurol 2015; 61: 529-35.

(c) 2015 Revista de Neurología 
Tabla I. Protocolo de evaluación neuropsicológica.

\begin{tabular}{|c|c|c|}
\hline \multirow{8}{*}{$\begin{array}{l}\text { Funciones } \\
\text { cognitivas }\end{array}$} & \multirow{2}{*}{$\begin{array}{l}\text { Memoria de } \\
\text { trabajo verbal }\end{array}$} & Dígitos directos e inversos: test Barcelona revisado [28] \\
\hline & & Letras y números: escala de memoria de Wechsler III [29] \\
\hline & $\begin{array}{l}\text { Memoria de } \\
\text { trabajo visual }\end{array}$ & $\begin{array}{l}\text { Localización espacial directa e inversa: } \\
\text { escala de memoria de Wechsler III [29] }\end{array}$ \\
\hline & Atención alternante & Trail Making Test $B$ [30] \\
\hline & Atención sostenida & $\begin{array}{l}\text { Conners' Performance Test II (CPT-II) [31]: omisiones, } \\
\text { comisiones, perseveraciones, detectabilidad y variabilidad }\end{array}$ \\
\hline & Habilidades & Cubos: test Barcelona revisado [28] \\
\hline & y visuoconstructivas & Test de retención visual de Benton, forma C [32] \\
\hline & Memoria & $\begin{array}{l}\text { Test de aprendizaje verbal España-Complutense (TAVEC) [33]: } \\
\text { recuerdo libre total, recuerdo libre a corto y largo plazo, } \\
\text { recuerdo con claves a corto y largo plazo, y reconocimiento }\end{array}$ \\
\hline \multirow{3}{*}{ Psicopatología } & \multicolumn{2}{|c|}{ Inventario de depresión de Beck II (BDI II) [34] } \\
\hline & \multicolumn{2}{|c|}{ Escala de ansiedad de Hamilton (HARS) [35] } \\
\hline & \multicolumn{2}{|c|}{ Symptom Checklist-90-revised (SCL-90) [36] } \\
\hline \multirow{2}{*}{$\begin{array}{l}\text { Nivel de } \\
\text { funcionalidad }\end{array}$} & \multicolumn{2}{|c|}{ Cuestionario de síntomas disejecutivos (DEX) [37] } \\
\hline & \multicolumn{2}{|c|}{ Interview for Deterioration in Daily Living Activities in Dementia (IDDD) [38] } \\
\hline
\end{tabular}

cionalidad [20]; también abulia, apatía y evitación social [21], y la ansiedad y la depresión son las patologías más frecuentes [22]. Otros estudios sugieren cambios en los rasgos de personalidad [23,24] y presencia de rasgos ansiosos de personalidad [25], específicamente evitativos [5], dependientes [12,26] obsesivo-compulsivos y pasivo-agresivos [5,23], frente a otros que destacan los rasgos de personalidad del cluster A, esquizotípicos [23,20], paranoides [12, 26] y agresivos [12]. El perfil de personalidad descrito se entiende como consecuencia de la afectación cerebral progresiva más que como resultado de un proceso adaptativo posterior al diagnóstico [23]. Como se ha mencionado más arriba, el estado cognitivo y emocional tiene un impacto negativo en la calidad de vida $[7,27]$, y por ello se considera imprescindible la evaluación neuropsicológica.

El objetivo de este artículo es describir el perfil cognitivo y psicopatológico de una muestra de pacientes con enfermedad de Steinert. Este trabajo se enmarca dentro de un proyecto de investigación en DM-1 en la que participan tres servicios del Complejo Hospitalario de Navarra: neurología, neurorradiología y neuropsicología.

\section{Pacientes y métodos}

Se empleó una muestra de 27 pacientes con diagnóstico de DM-1, variante clásica o de inicio adulto, en seguimiento en consultas externas del Servicio de Neurología del Complejo Hospitalario de Navarra. Los criterios de inclusión fueron tener menos de 50 años y descartar cualquier otra patología o condición física que impidiese realizar la evaluación neuropsicológica o las pruebas médicas que formaban parte del protocolo de seguimiento en esta patología, habiendo firmado previamente el consentimiento informado. El protocolo de pruebas, diseñado por un neuropsicólogo, incluyó los instrumentos de evaluación detallados en la tabla I [28-38].

\section{Resultados}

El perfil sociodemográfico de la muestra se detalla en la tabla II.

Desde el punto de vista psicopatológico, la muestra no presentó sintomatología depresiva ni ansiosa clínicamente significativa, de acuerdo con los criterios clínicos establecidos por los cuestionarios inventario de depresión de Beck [34] y escala de ansiedad de Hamilton [35]. Los dominios somáticos con puntuaciones significativamente más elevadas en el Symptom Checklist-90-revised [36] fueron ideación paranoide, depresión, obsesión-compulsión y sensibilidad interpersonal (Tabla III).

La evaluación neuropsicológica puso de manifiesto déficits en habilidades visuoconstructivas y visuoespaciales y atención alternante. En las pruebas de memoria de trabajo verbal [39], el grupo de pacientes tuvo un rendimiento medio-bajo en dígitos directos e inversos y localización espacial directa, con un percentil situado entre 19 y 28 [40]. En localización espacial inversa, el rendimiento fue menor, y la puntuación obtenida se corresponde con un percentil de $10 \mathrm{o}$ inferior [40]. La variabilidad en los datos de los índices seleccionados en el test de aprendizaje verbal España-Complutense (TAVEC) [33] como medida de memoria verbal a corto y largo plazo condicionó la interpretación.

En atención sostenida, las únicas puntuaciones bajas fueron en el índice variabilidad del Conners' Performance Test II [31]. El rendimiento en el Trail Making Test (TMT) B [30] como medida de atención alternante fue también bajo. La puntuación media (tiempo de ejecución) en esta última prueba se correspondió con un percentil inferior a 1 [40]; sin embargo, las dificultades psicomotrices características de la DM-1 podrían sesgar estos resultados. 
En habilidades visuoconstructivas y visuoespaciales, los pacientes cometieron errores en la copia de figuras del test de retención visual de Benton [32], y la puntuación en cubos (sin tiempo) [28] correspondió a un percentil inferior a 5 (Tabla IV).

El cuestionario de síntomas disejecutivos (DEX) [37] y la escala Interview for Deterioration in Daily Living Activities in Dementia (IDDD) [38] reflejaron dificultades ejecutivas en la vida diaria y en la autonomía funcional (no incluidas en las tablas). Partiendo de que no existen puntos de corte específicos en DM-1, las puntuaciones medias de ambos instrumentos fueron indicativas de probable deterioro ejecutivo según el DEX (media: $24,45 \pm 16,58$ ) [41] y superiores al punto de corte establecido para deterioro funcional en la IDDD (media: 41,05 \pm $13,95)$ [42]. Por último, la categorización 'con déficits cognitivos', elaborada a partir del análisis global del rendimiento en las pruebas y la información recogida en la historia clínica, permitió concluir que el $85,2 \%$ de los pacientes afectos de DM-1 presentó dichos déficits.

\section{Discusión}

La DM-1 es una enfermedad multisistémica y progresiva $[7,10,22]$ que precisa un abordaje multidisciplinar, en el que el neuropsicólogo es el profesional encargado de determinar el estado cognitivo y realizar el seguimiento de éste.

En este trabajo, el perfil cognitivo se obtuvo empleando un protocolo de evaluación neuropsicológica adecuado en cuanto al tiempo de evaluación requerido y con una fuerte carga de medida en funciones ejecutivas [43]. Algunas de estas funciones frontales o ejecutivas, como la memoria de trabajo, la flexibilidad cognitiva o el acceso a los contenidos de memoria, y otras características que condicionan un buen funcionamiento ejecutivo, como la velocidad de procesamiento, se han relacionado con una conducta inteligente [44]. No se empleó una medida estandarizada de cociente de inteligencia según los siguientes supuestos:

- Los tests de inteligencia valoran funciones muy dispares y que se relacionarían con diversas redes cerebrales [45].

- La puntuación total o cociente de inteligencia podría enmascarar en algunos casos déficits en algunos de los dominios cognitivos medidos.

- Varios de los trabajos revisados hacen referencia a un 'retraso intelectual', pero lejos de la conceptualización de cociente de inteligencia de Wechsler, y en la DM-1 no es frecuente hallar un dete-
Tabla II. Variables demográficas.

\begin{tabular}{|c|c|c|c|}
\hline & & $n$ & $\%$ \\
\hline \multicolumn{2}{|c|}{ Edad (media \pm desviación estándar) } & \multicolumn{2}{|c|}{$38,59 \pm 9,219$ años } \\
\hline \multirow{2}{*}{ Sexo } & Hombre & 13 & 48,1 \\
\hline & Mujer & 14 & 51,9 \\
\hline \multirow{4}{*}{ Nivel educativo } & Primarios & 8 & 29,6 \\
\hline & Secundarios/Formación profesional de grado medio & 17 & 63,0 \\
\hline & Formación profesional de grado superior & 0 & - \\
\hline & Universitarios & 2 & 7,4 \\
\hline \multirow{8}{*}{ Situación laboral actual } & Estudiante & 4 & 14,8 \\
\hline & Trabajador cualificado & 2 & 7,4 \\
\hline & Trabajador no cualificado & 5 & 18,5 \\
\hline & Trabajo protegido para personas con discapacidad & 1 & 3,7 \\
\hline & Desempleado & 3 & 11,1 \\
\hline & Trabajo doméstico & 1 & 3,7 \\
\hline & Incapacidad laboral permanente total & 1 & 3,7 \\
\hline & Incapacidad laboral permanente absoluta & 10 & 37,0 \\
\hline \multirow{2}{*}{ Situación laboral } & Activo & 16 & 59,3 \\
\hline & Incapacidad laboral & 11 & 40,7 \\
\hline \multirow{7}{*}{$\begin{array}{l}\text { Antecedentes personales } \\
\text { psiquiátricos/discapacidad } \\
\text { intelectual }\end{array}$} & Ausencia de antecedentes personales de interés & 17 & 63,0 \\
\hline & Trastorno depresivo mayor & 1 & 3,7 \\
\hline & Trastorno de la personalidad & 0 & - \\
\hline & Trastorno ansioso-depresivo & 4 & 14,8 \\
\hline & Discapacidad intelectual & 3 & 11,1 \\
\hline & Trastorno adaptativo & 1 & 3,7 \\
\hline & Trastorno por déficit de atención/hiperactividad & 1 & 3,7 \\
\hline \multicolumn{2}{|c|}{ Ausencia de antecedentes psiquiátricos/discapacidad intelectual } & 17 & 63,0 \\
\hline
\end{tabular}

rioro cognitivo global, sino limitado a determinadas funciones $[15,24,46]$.

- Resultaría discutible aplicar una medida de inteligencia premórbida una vez realizado el diagnóstico (dado que el diagnóstico de DM-1 suele 
Tabla III. Variables psicopatológicas (media £ desviación estándar).

\begin{tabular}{|c|c|c|}
\hline \multicolumn{2}{|c|}{ Inventario de depresión de Beck II } & $12,04 \pm 9,70$ \\
\hline \multicolumn{2}{|c|}{ Escala de ansiedad de Hamilton } & $13,16 \pm 9,23$ \\
\hline \multirow{13}{*}{$\begin{array}{l}\text { Symptom } \\
\text { Checklist- } \\
\text { 90-revised }\end{array}$} & Índice global de gravedad & $0,61 \pm 0,45$ \\
\hline & Número total de síntomas positivos & $23,00 \pm 0,00$ \\
\hline & Intensidad total de síntomas & $0,07 \pm 0,16$ \\
\hline & Somatización & $0,33 \pm 0,00$ \\
\hline & Obsesión-compulsión & $0,84 \pm 0,52$ \\
\hline & Sensibilidad interpersonal & $0,80 \pm 0,65$ \\
\hline & Depresión & $0,88 \pm 0,66$ \\
\hline & Ansiedad & $0,46 \pm 0,51$ \\
\hline & Hostilidad & $0,38 \pm 0,48$ \\
\hline & Ansiedad fóbica & $0,29 \pm 0,35$ \\
\hline & Ideación paranoide & $4,08 \pm 17,28$ \\
\hline & Psicoticismo & $0,50 \pm 0,70$ \\
\hline & Síntomas discretos & $4,24 \pm 17,05$ \\
\hline
\end{tabular}

realizarse de forma tardía y que los déficits serían resultado de una afectación que lleva años produciéndose).

- Las dificultades visuales y visuoperceptivas de estos pacientes impiden aplicar pruebas estándares con carga visual.

La bibliografía revisada mostró cierto consenso en los dominios cognitivos evaluados, pero variabilidad en los protocolos de evaluación neuropsicológica, el tamaño muestral, el empleo de muestra control y la determinación de afectación cognitiva. Comenzando por la memoria verbal a corto y largo plazo, varios trabajos que emplean pruebas similares al TAVEC [33] no encuentran diferencias significativas respecto al rendimiento de un grupo control $[1,9,13]$, mientras que otros autores sí describen resultados inferiores tras una única evaluación $[8,12$, 46] o en una segunda evaluación [7].

El bajo rendimiento en span de dígitos y localización espacial (memoria de trabajo), con puntuaciones inferiores a los datos normativos españoles, corroboran los hallazgos de varios autores [7,12,13,24], mientras que otros trabajos no encuentran diferen- cias significativas en el span de dígitos a la hora de compararlo con un grupo control [14], así como tampoco en una segunda evaluación $[8,9]$. Los resultados en habilidades visuoconstructivas y visuoespaciales son también opuestos. En el subtest de cubos [28] obtienen resultados inferiores a los descritos por otros autores que emplean también esta prueba $[1,12,14]$, la copia de figuras simples o la figura compleja de Rey [1,12-14,16,27], aunque en todos estos trabajos la variabilidad en la composición muestral hace que no esté exenta de sesgo esta comparación. Concretamente, Zalonis et al no encuentran un rendimiento inferior en el diseño de bloques [14] y otros autores únicamente lo describen al comparar al grupo de DM-1 con el de DM-2 [10,47].

Además del diseño de bloques, otra de las pruebas más empleadas y que se considera fundamental en los protocolos de evaluación en la DM-1 es el TMT [48]. Los resultados de nuestra muestra ponen de manifiesto un rango de puntuaciones equivalentes a un percentil 30 e inferior [40], inferiores a las alcanzadas en algunos trabajos $[8,13,24]$ y similares a las descritas en otro trabajo [16]. Una vez más, otros autores no encuentran resultados inferiores en el TMT-B en comparación con grupos controles $[14,15,49]$. Frente al empleo mayoritario del TMT como medida atencional-ejecutiva, son pocos los trabajos revisados que empleen tareas de ejecución continua. Sistiaga et al señalan el rendimiento inferior del grupo con DM-1 [12], pero no otros trabajos que emplean medidas atencionales diferentes [5-49]. Esta variabilidad en los resultados destaca la importancia de estandarizar los protocolos, definir con mayor exactitud los déficits y establecer pautas de intervención $[43,48]$.

En este trabajo se empleó una de las escalas más usadas en la evaluación de la funcionalidad en la demencia [37] y, en esta línea, otros autores emplean otras escalas de cribado cognitivo en demencias, como el Addenbrooke's Cognitive Examinationrevised y el Minimental State Examination [5,6,8,9, $11,13,20,46,50]$. La presencia de alteraciones neuroanatómicas similares a las encontradas en las demencias y la afectación cognitiva multidominio son factores que hacen que el seguimiento neuropsicológico de estos pacientes sea imprescindible.

En psicopatología, la muestra obtuvo únicamente un rango de puntuaciones elevadas en depresión, al igual que han encontrado otros autores [3,11], y niveles superiores de sufrimiento psíquico y psicosomático global. En relación con variables de personalidad, presentaron puntuaciones elevadas en obsesión-compulsión, sensibilidad interpersonal, ideación paranoide y psicoticismo. Este perfil de rasgos 
ansiosos y del espectro psicótico es similar al encontrado por otros autores [5,20,23]. Mientras que algunos trabajos defienden que la psicopatología es resultado de un proceso de adaptación a la enfermedad [17], otros destacan que los cambios patológicos cerebrales serían los responsables directos de su estado anímico $[5,16]$.

La necesidad de incluir en los protocolos de evaluación medidas de cognición social (reconocimiento facial de emociones, memoria facial, sociabilidad) se puso de manifiesto en la reciente Outcome Measures in Myotonic Dystrophy Type 1 [43]. Algunos trabajos han demostrado déficits en memoria facial [1], deterioro en cognición social y baja sociabilidad [17,19], y en el reconocimiento de expresiones negativas faciales [19]; con dificultad para entender los estados mentales de las otras personas a partir de sus interacciones y de las expresiones faciales, menor sensibilidad a reconocer observaciones inapropiadas y el impacto emocional en los otros. Los rasgos de personalidad antes descritos podrían estar relacionados con los déficits en cognición social $[12,17,23]$, pero otra posible hipótesis sería la de que la hipotonía facial frecuente en estos pacientes no les permitiría 'imitar' la expresión que están observando, dificultando así el poder interpretarla.

Síntomas característicos de la DM-1, como la afectación muscular progresiva, la fatiga, la hipersomnolencia o el deterioro cognitivo, entre otros, merman el nivel de autonomía y repercuten en las actividades de la vida diaria, en el ámbito laboral y social $[7,11]$. La evaluación de la calidad de vida es, por tanto, imprescindible en estos pacientes $[7,43$, 48]. Si bien la mayoría de los trabajos emplean escalas de calidad de vida como la Short-Form 36, el Individualized Neuromuscular Quality of Life Questionnaire o la Menopause Rating Scale [11,14,20,26, 48], la interpretación cualitativa de la escala empleada en este trabajo, la IDDD [37], puso de manifiesto que la mayor parte de los pacientes presenta dificultades en actividades básicas e instrumentales de la vida diaria en función del nivel de gravedad y progresión de la patología, afectación corroborada por los resultados del cuestionario DEX [38]. Se empleó la versión familiar, dado que el avance de la enfermedad puede acompañarse de un menor insight [49].

Por último, hay que ser cautos a la hora de extrapolar los resultados de esta investigación. Por un lado, se trata de una muestra relativamente pequeña $y$, dada la singularidad de los pacientes, no se ha creído pertinente contar con un grupo de control. Por otro lado, se ha encontrado una alta variabili-
Tabla IV. Variables neuropsicológicas (media \pm desviación estándar).

\begin{tabular}{|c|c|c|}
\hline \multirow{3}{*}{$\begin{array}{l}\text { Memoria de } \\
\text { trabajo verbal }\end{array}$} & Dígitos directos & $5,41 \pm 1,39$ \\
\hline & Dígitos inversos & $4,22 \pm 1,31$ \\
\hline & Letras y números & $4,37 \pm 1,50$ \\
\hline \multirow{2}{*}{$\begin{array}{l}\text { Memoria de } \\
\text { trabajo visual }\end{array}$} & Localización espacial directa & $5,11 \pm 1,67$ \\
\hline & Localización espacial indirecta & $4,37 \pm 0,84$ \\
\hline \multirow{3}{*}{$\begin{array}{l}\text { Habilidades } \\
\text { visuoconstructivas } \\
\text { y visuoespaciales }\end{array}$} & Test de retención visual de Benton & $7,78 \pm 3,03$ \\
\hline & Cubos & $3,15 \pm 1,68$ \\
\hline & Cubos tiempo & $7,67 \pm 4,09$ \\
\hline \multirow{5}{*}{ Atención sostenida } & Omisiones & $58,79 \pm 19,59$ \\
\hline & Comisiones & $50,20 \pm 11,39$ \\
\hline & Perseveraciones & $67,41 \pm 33,83$ \\
\hline & Detectabilidad & $47,81 \pm 9,70$ \\
\hline & Variabilidad & $57,51 \pm 10,19$ \\
\hline \multicolumn{2}{|l|}{ Trail Making Test $B$} & $169,78 \pm 103,75$ \\
\hline \multirow{6}{*}{ Memoria verbal } & Recuerdo libre total & $-0,26 \pm 1,06$ \\
\hline & Recuerdo libre a corto plazo & $-0,19 \pm 0,83$ \\
\hline & Recuerdo con claves a corto plazo & $-0,33 \pm 1,04$ \\
\hline & Recuerdo libre a largo plazo & $-0,04 \pm 1,19$ \\
\hline & Recuerdo con claves a largo plazo & $-0,26 \pm 1,13$ \\
\hline & Reconocimiento & $0,22 \pm 1,15$ \\
\hline \multirow{2}{*}{$\begin{array}{l}\text { Diagnóstico } \\
\text { neuropsicológico }\end{array}$} & Sin déficits cognitivos & $4(14,8 \%)$ \\
\hline & $\begin{array}{l}\text { Deterioro cognitivo leve unidominio, multidominio } \\
\text { o demencia en enfermedad de Steinert }\end{array}$ & $23(85,2 \%)$ \\
\hline
\end{tabular}

dad en los resultados de algunas pruebas, aunque esta variabilidad puede reflejar la propia idiosincrasia de la enfermedad. En este sentido, es necesario continuar con la línea de investigación comenzada y realizar un seguimiento cognitivo y psicopatológico de los pacientes con DM-1. También sería conveniente incluir la evaluación de algunas áreas, como el procesamiento facial y el de las emociones [ 1 , 18-20], o una evaluación de variables de personalidad. Todas estas medidas permitirán valorar la hipótesis planteada por algunos autores sobre el desarrollo de posible demencia en pacientes con DM-1. 
Bibliografía

1. Kleberg JL, Lindberg C, Winblad S. Facial memory deficits in myotonic dystrophy type 1 . Acta Neurol Scand 2014; 130: 312-8.

2. Romeo V. Myotonic dystrophy type 1 or Steinert's disease. Adv Exp Med Biol 2012; 724: 239-57.

3. Franzese A, Antonini G, Iannelli M, Leardi MG, Spada S, Vichi R, et al. Intellectual functions and personality in subjects with noncongenital myotonic muscular dystrophy. Psychol Rep 1991; 68: 723-32.

4. Ashizawa T, Sarkar PS. Myotonic dystrophy types 1 and 2. In Griggs RC, Amato AA, eds. Handbook of clinical neurology. Vol. 101. London: Elsevier; 2011. p. 193-237.

5. Meola G, Sansone V, Perani D, Scarone S, Cappa S, Dragoni C et al. Executive dysfunction and avoidant personality trait in myotonic dystrophy type 1 (DM-1) and in proximal myotonic myopathy (PROMM/DM-2). Neuromuscul Disord 2003; 13 813-21.

6. Modoni A, Silvestri G, Pomponi MG, Mangiola F, Tonali P, Marra C. Characterization of the pattern of cognitive impairment in myotonic dystrophy type 1. Arch Neurol 2004; 61: 1943-7.

7. Antonini G, Soscia F, Giubilei F, De Carolis A, Gragnani F, Morino S, et al. Health-related quality of life in myotonic dystrophy type 1 and its relationship with cognitive and emotional functioning. J Rehabil Med 2006; 38: 181-5.

8. Sansone V, Gandossini S, Cotelli M, Calabria M, Zanetti O, Meola G. Cognitive impairment in adult myotonic dystrophies: a longitudinal study. Neurol Sci 2007; 28: 9-15.

9. Modoni A, Silvestri G, Vita MG, Quaranta D, Tonali P, Marra C. Cognitive impairment in myotonic dystrophy type 1 (DM1) a longitudinal follow-up study. J Neurol 2008; 255: 1737-42.

10. Romeo V, Pegoraro E, Ferrati C, Squarzanti F, Sorarù G, Palmieri A, et al. Brain involvement in myotonic dystrophies: neuroimaging and neuropsychological comparative study in DM1 and DM2. J Neurol 2010; 257: 1246-55.

11. Rakocevic-Stojanovic V, Peric S, Madzarevic R, Dobricic V, Ralic V, Ilic V, et al. Significant impact of behavioral and cognitive impairment on quality of life in patients with myotonic dystrophy type 1. Clin Neurol Neurosurg 2014; 126: 76-81.

12. Sistiaga A, Urreta I, Jodar M, Cobo AM, Emparanza J Otaegui D, et al. Cognitive/personality pattern and triplet expansion size in adult myotonic dystrophy type 1 (DM1): CTG repeats, cognition and personality in DM1. Psychol Med 2010; 40: 487-95.

13. Weber YG, Roebling R, Kassubek J, Hoffmann S, Rosenbohm A Wolf $\mathrm{M}$, et al. Comparative analysis of brain structure, metabolism, and cognition in myotonic dystrophy 1 and 2 . Neurology 2010; 74: 1108-17.

14. Zalonis I, Bonakis A, Christidi F, Vagiakis E, Papageorgiou SG, Kalfakis N, et al. Toward understanding cognitive impairment in patients with myotonic dystrophy type 1 . Arch Clin Neuropsychol 2010; 25: 303-13.

15. Tanaka H, Arai M, Harada M, Hozumi A, Hirata K. Cognition and event-related potentials in adult-onset non-demented myotonic dystrophy type 1. Clin Neurophysiol 2012; 123: 261-9.

16. Caso F, Agosta F, Peric S, Rakočević-Stojanović V, Copetti M, Kostic VS, et al. Cognitive impairment in myotonic dystrophy type 1 is associated with white matter damage. PLoS One 2014; 9: e104697.

17. Winblad S, Hellström P, Lindberg C, Hansen S. Facial emotion recognition in myotonic dystrophy type 1 correlates with CTG repeat expansion. J Neurol Neurosurg Psychiatry 2006; 77: 219-23.

18. Kobayakawa M, Tsuruya N, Takeda A, Suzuki A, Kawamura M Facial emotion recognition and cerebral white matter lesions in myotonic dystrophy type 1. J Neurol Sci 2010; 290: 48-51.

19. Kobayakawa M, Tsuruya N, Kawamura M. Theory of mind impairment in adult-onset myotonic dystrophy type 1. Neurosci Res 2012; 72: 341-6.

20. Serra L, Silvestri G, Petrucci A, Basile B, Masciullo M Makovac E, et al. Abnormal functional brain connectivity and personality traits in myotonic dystrophy type 1. JAMA Neurol 2014; 71: 603-11.
21. D'Angelo MG, Bresolin N. Cognitive impairment in neuromuscular disorders. Muscle Nerve 2006; 34: 16-33

22. Turner $\mathrm{C}$, Hilton-Jones D. The myotonic dystrophies: diagnosis and management. J Neurol Neurosurg Psychiatry 2010; 81 358-67.

23. Delaporte C. Personality patterns in patients with myotonic dystrophy. Arch Neurol 1998; 55: 635-40.

24. Winblad S, Lindberg C, Hansen S. Cognitive deficits and CTG repeat expansion size in classical myotonic dystrophy type 1 (DM1). Behav Brain Funct 2006; 2: 16.

25. Laberge L, Mathieu J, Auclair J, Gagnon E, Noreau L, Gagnon C. Clinical, psychosocial, and central correlates of quality of life in myotonic dystrophy type 1 patients. Eur Neurol 2013; 70: 308-15.

26. Peric S, Sreckov M, Basta I, Lavrnic D, Vujnic M, Marjanovic I, et al. Dependent and paranoid personality patterns in myotonic dystrophy type 1. Acta Neurol Scand 2014; 129: 219-25.

27. Nätterlund B, Gunnarsson LG, Ahlström G. Disability, coping and quality of life in individuals with muscular dystrophy: a prospective study over five years. Disabil Rehabil 2000; 22: 776-85.

28. Peña-Casanova J. Test Barcelona revisado. Barcelona: Masson; 2005.

29. Wechsler D. Escala de memoria para adultos, tercera edición. Madrid: TEA Ediciones; 1999.

30. Horton AM, Hartlage LC. The Halstead-Reitan neuropsychology test battery: theory and clinical interpretation, second edition. Arch Clin Neuropsychol 1994; 9: 289-90.

31. Conners CK. Continuous Perfomance Test, second edition. New York: MHS; 1994.

32. Benton AL. TRVB: test de retención visual de Benton. Madrid: TEA Ediciones; 2011.

33. Benedet MI, Alejandre MA. TAVEC: test de aprendizaje verbal España-Compultense. Madrid: TEA Ediciones; 1999.

34. Beck AT, Steer RA, Brown GK. BDI-II: inventario de depresión de Beck II. Madrid: Pearson; 2011.

35. Hamilton $M$. The assessment of anxiety states by rating. Br J Med Psychol 1959; 32: 50-5.

36. Derogatis LR. SCL-90-R. Cuestionario de 90 síntomas. Madrid: TEA Ediciones; 2002.

37. Wilson BA, Aldermam N, Burguess PW. The behavioural assessment of dysexecutive syndrome. London: Pearson; 1996.

38. Teunisse S, Derix MM, Crevel H. Assessing the severity of dementia. Patient and caregiver. Arch Neurol 1991; 48: 274-7.

39. Blázquez-Alisente JL, González-Rodríguez B, Paúl-Lapedriza N. Evaluación neuropsicológica. In Tirapu-Ustárroz J, RíosLago M, Maestú-Unturbe F, eds. Manual de neuropsicología. 2 ed. Barcelona: Viguera; 2011. p. 35-56.

40. Tamayo F, Casals-Coll M, Sánchez-Benavides G, Quintana M, Manero RM, Rognoni T, et al. Estudios normativos españoles en población adulta joven (proyecto NEURONORMA jóvenes): normas para las pruebas span verbal, span visuoespacial, Letter-Number Sequencing, Trail Making Test y Symbol Digit Modalities Test. Neurologia 2012; 27: 319-29.

41. Llanero-Luque M, Ruiz-Sánchez de León JM, Pedrero-Pérez EJ, Olivar-Arroyo A, Bouso-Saiz JC, Rojo-Mota G, et al. Sintomatología disejecutiva en adictos a sustancias en tratamiento mediante la versión española del cuestionario disejecutivo (DEX-Sp). Rev Neurol 2008; 47: 457-63.

42. Böhm P, Peña-Casanova J, Aguilar M, Hernández G, Sol JM, Blesa R. Clinical validity and utility of the interview for deterioration of daily living in dementia for Spanish-speaking communities. Int Psychogeriatr 1998; 10: 261-70.

43. Gagnon C, Meola G, Hébert LJ, Laberge L, Leone M, Heatwole C. Report of the second Outcome Measures in Myotonic Dystrophy type 1 (OMMYD-2). Neuromuscul Disord 2015; 25: 603-16.

44. García-Molina A, Tirapu-Ustárroz J, Luna-Lario P, Ibañez J, Duque P. ¿Son lo mismo inteligencia y funciones ejecutivas? Rev Neurol 2010; 50: 738-46.

45. Luna-Lario P, Tirapu-Ustárroz J, Ibáñez-Alonso J, GarcíaMolina A, Duque P. Inteligencia y funciones ejecutivas. In Tirapu-Ustárroz J, García-Molina A, Ríos-Lago M, Ardila- 
Ardila A, eds. Neuropsicología de la corteza prefrontal y las funciones ejecutivas. Barcelona: Viguera; 2012. p. 327-50.

46. Rubinsztein JS, Rubinsztein DC, McKenna PJ, Goodburn S, Holland AJ. Mild myotonic dystrophy is associated with memory impairment in the context of normal general intelligence. J Med Genet 1997; 34: 229-33.

47. Gaul C, Schmidt T, Windisch G, Wieser T, Müller T, Vielhaber S, et al. Subtle cognitive dysfunction in adult onset myotonic dystrophy type 1 (DM1) and type 2 (DM2). Neurology 2006; 67: 350-2.

48. Axford MM, Pearson CE. Illuminating CNS and cognitive issues in myotonic dystrophy: workshop report. Neuromuscul Disord 2013; 23: 370-4.

49. Minnerop M, Weber B, Schoene-Bake JC, Roeske S, Mirbach S, Anspach C, et al. The brain in myotonic dystrophy 1 and 2: evidence for a predominant white matter disease. Brain 2011; 134: $3530-46$

50. Caliandro P, Silvestri G, Padua L, Bianchi MLE, Simbolotti C, Russo G, et al. fNIRS evaluation during a phonemic verbal task reveals prefrontal hypometabolism in patients affected by myotonic dystrophy type 1 . Clin Neurophysiol 2013; 124 : 2269-76.

\section{A descriptive study of the neuropsychological and psychopathological profile in patients with type 1 myotonic dystrophy}

Introduction. Type 1 myotonic dystrophy (MD-1) or Steinert disease is a multisystemic progressive disorder. Studies have shown cognitive deficits, depressive symptoms and a high incidence of anxiety personality traits that compromise both the functionality and the quality of life of these patients.

Aim. To describe the cognitive and psychopathological profile of a sample of patients with the adult variant of MD-1.

Patients and methods. A sample of 27 patients diagnosed with MD-1 was selected from those being followed up in the neurology service of the Complejo Hospitalario de Navarra. Eligibility criteria were age under 50 years and the absence of any other pathology or physical condition that prevented them from performing the psychological evaluation. A neuropsychological evaluation battery specifically designed for this kind of pathology was used, together with psychopathological and functionality measures.

Results. The neuropsychological evaluation revealed mainly deficits in visual-constructional and visuospatial skills, alternating attention and in other-reported dysexecutive signs and symptoms. The group of patients did not present any clinically significant symptoms of depression or anxiety, but did score high on obsession-compulsion, interpersonal sensitivity, paranoid ideation and psychoticism. The results point towards a deterioration in functionality.

Conclusions. In the integral approach to MD-1, the characterisation and developmental monitoring of the cognitive, psychopathological and personality profile, as well as the level of functionality, all contribute to an improvement in the quality of life of these patients.

Key words. Cognitive functioning. Dysexecutive syndrome. Emotional status. Myotonic dystrophy. Personality. Steinert disease. 